\title{
République Centrafricaine. Musiques des anciennes cours Bandia
}

Le Chant du Monde, Paris, 1996

\section{Nathalie Fernando}

\section{OpenEdition}

\section{Journals}

Édition électronique

URL : http://journals.openedition.org/ethnomusicologie/944

ISSN : 2235-7688

Éditeur

ADEM - Ateliers d'ethnomusicologie

Édition imprimée

Date de publication : 1 décembre 1997

Pagination : 361-365

ISBN : 2-8257-0579-9

ISSN : 1662-372X

Référence électronique

Nathalie Fernando, "République Centrafricaine. Musiques des anciennes cours Bandia », Cahiers d'ethnomusicologie [En ligne], 10 | 1997, mis en ligne le 06 janvier 2012, consulté le 19 avril 2019. URL: http://journals.openedition.org/ethnomusicologie/944

Ce document a été généré automatiquement le 19 avril 2019

Tous droits réservés 


\title{
République Centrafricaine. Musiques des anciennes cours Bandia
}

\author{
Le Chant du Monde, Paris, 1996
}

Nathalie Fernando

\section{RÉFÉRENCE}

République Centrafricaine. Musiques des anciennes cours Bandia. Enregistrements, textes et photos de Eric de Dampierre et Marc Chemillier ; traduction anglaise : Peter Crowe. Livret bilingue français/anglais de 127 pages, carte, croquis d'instruments, transcriptions, traduction des chants - à l'exception des plages 13, 14, 15 - ainsi que sept photos. 1 CD Le Chant du Monde 274 1009, collection C.N.R.S./ Musée de l'Homme, Paris 1996.

1 Ce disque, consacré aux musiques des anciennes cours Bandia, réunit des enregistrements collectés chez les Nzakara et les Zandé par Eric de Dampierre - dans les années 1965 à 1985 (13 plages) et Marc Chemillier - en 1993 (5 plages). Il propose un éventail instrumental et vocal représentatif de la musique de ces deux ethnies, une place toute particulière étant accordée à l'art des anciens poètes-harpistes nzakara.

2 Les Nzakara et les Zandé forment une population d'environ un demi-million d'individus, répartie sur la zone frontalière entre la République Centrafricaine, le Zaïre et le Soudan. Au siècle dernier, ils constituaient neufs royaumes : six, dans la partie Est, vivaient sous l'autorité Vungara - puissant clan autochtone qui s'était allié aux Zandé dont ils favorisèrent l'expansion ; les trois autres étaient placés sous la domination du clan Bandia - d'origine Ngbandi - qui conquit la partie Ouest du territoire zandé, ainsi que le pays nzakara dont il adopta la langue et nombre de coutumes. Les langues nzakara et zandé sont apparentées: la première était parlée dans l'un des trois royaumes bandia, la seconde, dans tous les autres royaumes. Autrefois, les pratiques musicales des deux ethnies étaient fortement liées à la vie de cour, mais force est de constater qu'à l'heure 
actuelle, la disparition progressive des structures qui régissaient l'organisation politique et sociale de ces royaumes a grandement contribué à la ruine de leur patrimoine culturel.

3 Les Nzakara possèdent un certain nombre de termes pour classer l'ensemble de leur musique tels que ngbàkià, limanza, nakùlúngù, lịkutạ, gitangi, gbàyà et kele-gbe ${ }^{n} l e$ (Chemillier 1995 : 123). Chacun d'eux fait référence à une danse traditionnelle en même temps qu'il désigne une catégorie musicale déterminée par des critères musicaux : les danses ngbàkià (danse royale exécutée lors de la fête des ancêtres, héritée des anciens lignages Voukpata), limanza (effectuée lorsque le chef boit ou fume et rythmée par les frappements de mains de l'assistance) et nakùlúngù (dansée au lever du roi, exclusivement réservée aux femmes et accompagnée de chants responsoriaux) sont toutes trois spécifiquement bandia. Gitangi, jeu dansé, et likutạ, associée à la consommation de chanvre, ne sont pas spécifiques aux Bandia. Enfin kele-gbe lé, pratiquée indifféremment par les hommes et les femmes, ainsi que gbàyà, réservée aux hommes, sont des danses de divertissement nzakara d'apparition récente.

Les pièces instrumentales nzakara présentées dans ce disque ne comportent donc pas de titre qui leur soit propre : elles sont désignées par le nom du répertoire de danse auquel elles sont empruntées ; la danse kele-gben le fait l'objet d'une exécution à la sanza (plage 13) tandis que les danses ngbàkià, limanza, gitangi sont jouées à la harpe (plages 1 à 8 ).

5 La harpe nzakara, couramment appelée kùndì, possède cinq cordes convergentes à la table d'harmonie. Sur les plus beaux instruments, une tête sculptée orne l'extrémité du manche ${ }^{1}$. Les cordes sont réparties entre les mains du musicien de la façon suivante : les deux cordes les plus aiguës et les plus graves sont pincées respectivement par le pouce et l'index de la main droite et de la main gauche, la corde centrale étant alternativement pincée par l'index de chacune d'elles.

6 Les pièces issues de la catégorie gitangi (plages $1,2,3$ et 6) se caractérisent par une formule rythmique répondant au procédé d'imparité déjà mis en évidence dans une grande partie des musiques d'Afrique centrale :

\section{D. . soit $[2+2+3 / 2+3]$}

7 Cette formule est matérialisée par la percussion de l'annulaire droit sur la peau qui recouvre la caisse de résonance mais peut également être directement intégrée au jeu polyphonique de la harpe (cf. plage 3). Par ailleurs, elle donne lieu à des variations modifiant le mode d'organisation du regroupement des durées minimales (cf. plage 1 où la formule proposée est $[2+2+2+3+3]$ ). Plage 2, l'instrumentiste fait preuve d'une très grande dextérité en combinant adroitement différentes superpositions de ce rythme entre doigt percuté et jeu mélodico-rythmique de la harpe, engendrant ainsi une polyrythmie complexe.

8 Cette formule rythmique asymétrique, caractéristique des pièces gitangi jouées à la harpe, apparait également dans la partie de grelots qui accompagne le chant nakùlúngù exécuté par un chœur de femmes et une soliste (plage 12). Le livret ne fournit cependant pas d'élément de réponse quant à une éventuelle filiation des deux catégories².

Les pièces des catégories ngbàkià et limanza donnent lieu à une exécution homorythmique peu variée faisant appel à cinq consonances différentes. La modalité de subdivision de la pulsation constitue l'élément qui permet de distinguer ces deux catégories: les pièces ngbàkià sont binaires alors que les pièces limanza - dont la pulsation lente est généralement soulignée par des frappements de mains de l'assistance - sont ternaires. 
Enfin, celles dont le cycle compte cinq ou dix pulsations ont la particularité d'être construites selon un procédé « en canon » qui s'établit entre les deux mains.

10 La présentation successive des huit pièces de harpe au début du disque pourrait lasser l'auditeur. Cependant, l'accord de l'instrument, qui diffère de plage en plage, renouvelle son attention et suscite nécessairement sa curiosité car le système pentatonique usité offre de multiples configurations. On constate, par exemple, que les échelles destinées à interpréter les pièces gitangi comportent des demi-tons et des quintes diminuées (plages 1 et 2) ou encore une succession de tons ${ }^{3}$ (plage 6). L'accord entre les deux harpes (plage 8) marque une différence pouvant aller jusqu'à vingt cents : ne s'agit-il pas ici d'une volonté délibérée de la part des musiciens d'enrichir le timbre en différenciant légèrement l'accord d'un même degré, plutôt que d'un désaccord pendant l'exécution, ainsi que le suggèrent les auteurs ? Par ailleurs, soulignons que, plage 4, la différence d'accord entre les deux harpes semble encore plus prononcée, mais les mesures des grandeurs d'intervalles ne sont pas précisées.

11 La harpe, « instrument de la vie civile ", revêt un caractère particulier lorsqu'elle se glisse entre les mains d'un poète-harpiste nzakara. Ce dernier, spécialiste mais non professionnel, n'a rien de commun avec un griot. Il n'est pas attaché à une cour et chante pour un public restreint, davantage captivé par sa parole que par sa musique. Mémoire vivante de l'histoire et en même temps témoin de son temps, sa parole est écoutée avec respect, plaisir ou crainte. Critique acerbe, il s'élève contre l'injustice (cf. «L'éléphant de la colère ", plage 7) ou se moque avec humour de la cour passive et fainéante (plage 4). La musique est là pour sublimer la parole qui, à son tour, se veut musique: plage 1, l'intonation de la voix se laisse porter par les tons et les tensions vocaliques de la langue, son débit rapide semblant mêler son propre rythme à celui du gitangi; au milieu de sa prestation, le musicien laisse libre cours à sa fantaisie et à plusieurs reprises, ses bruits de gorge et ses roulements de langue illustrent des propos empreints à la fois de tradition et de modernité (complainte faisant allusion au père qui s'éloigne sur son grillon comprendre par là, son Vélosolex). Le poète-harpiste manie avec aisance l'art de la rhétorique, utilisant nombre de métaphores et une mise en apposition systématique d'interjections. Par le biais d'un jeu subtil de substitutions, il évoque (plage 4) les tourments d'une vie sous l'autorité de "l'étranger», en la comparant aux douleurs causées par l'absorption d'un alcool fort «ce tord-boyaux, vous les Vou-gbandi, vous devez apprendre à le supporter pour continuer [à boire] ».

12 Outre des plages consacrées à la harpe, ce disque propose des pièces pour xylophone sur troncs de bananier ${ }^{4}$, pour xylophone portatif, pour sanza ainsi que des chants féminins, exécutés en solo a cappella ou en formation soliste/chœur accompagnée d'instruments rythmiques (grelots, cloches, tape-cuisses).

13 Le xylophone kpáníngbá est un instrument zandé pratiqué également chez les Nzakara. Son clavier comporte dix à quinze lames maintenues perpendiculairement à deux troncs de bananiers à l'aide de petits bâtonnets fichés dans chacun d'eux. Deux ou trois instrumentistes se placent côte à côte ou de part et d'autre du clavier, tenant une baguette dans chaque main; ce xylophone sur troncs de bananier, accompagné par un tambour à fente et des grelots (plage 18), anime les soirées de divertissement, les veillées mortuaires ou la fête des moissons. La sanza (plage 13) possède quant à elle un charme grésillant très discret: l'instrumentiste interprète ici une pièce dont le rythme ternaire, alternant longue/brève, est caractéristique du kele-gbe $e^{n} l e$. 
14 Par ailleurs, Eric de Dampierre et Marc Chemillier ont également tenu à nous faire entendre des instruments qui ont totalement disparu de nos jours : c'est le cas du xylophone portatif (plage 16) et des cloches doubles (plages 14 et 15). Le xylophone portatif qui pouvait être joué par les chefs ou le roi lui-même, accompagnait la danse royale ngbàkià. Contrairement à tous les xylophones de ce type que l'on rencontre en Afrique centrale, celui-ci surprend par son timbre métallique et par l'absence de mirliton à l'extrémité des calebasses qui font office de résonateurs ${ }^{5}$. Quant aux cloches doubles dont l'usage était exclusivement réservé aux femmes de la cour, elles se joignaient au jeu de tape-cuisses (plages 14 et 15), instrument nzakara au son curieux, rarissime dans ces royaumes.

Les plages 10 et 11 nous permettent d'apprécier la voix émouvante de Wolo, femme nzakara qui chante a cappella lors des veillées au coin du feu. Enfin, de sa voix féminine étrangement travestie, Batie nous conte la devise de l'oiseau ísò dans une chantefable où alternent voix parlée et voix chantée (plage 9).

Les enregistrements effectués à différentes époques nous permettent de porter un regard diachronique sur la musique des Nzakara-Zandé. En 1993, Marc Chemillier se heurta à de grandes difficultés et ne put reconstituer certaines formations instrumentales dans leur intégralité en raison de l'abandon progressif de la pratique de certains instruments. Les plages 17 et 18 nous rassurent en revanche quant au kpáníngbá : on constate avec plaisir que dans l'enregistrement de 1993, les musiciens font montre de la même vigueur et de la même dextérité que ceux qui participèrent à l'enregistrement réalisé vingt-cinq ans plus tôt, en particulier par la brillante utilisation de la technique du jeu alterné dans l'exécution de variations par monnayage.

L'ensemble de l'ouvrage témoigne d'une démarche esthétique visant à rendre compte non seulement de la réalité musicale, mais aussi du contexte sonore et de l'atmosphère poétique qui l'entourent : par instants, chants d'oiseaux et manifestations de l'assistance se mêlent à la musique. De plus, les enregistrements d'Eric de Dampierre représentent de précieux témoignages ; peu importe alors que la prise de son soit monophonique !

Enfin, le travail accompli par les deux auteurs constitue une réponse aux propos toujours d'actualité - que formulait Erich von Hornbostel il y a une soixantaine d'années :

19 «Jusqu'ici, nous savions à peine ce qu'est la musique africaine ; si nous ne nous hâtons pas de l'enregistrer au moyen du phonographe, nous ne saurons pas même ce qu'elle fut » (Hornbostel 1928 : 60).

\section{BIBLIOGRAPHIE}

AROM Simha, 1985, Polyphonies et polyrythmies d'Afrique centrale. Structure et méthodologie. Paris : SELAF.

CHEMILLIER Marc, 1995, " La musique de la harpe », in : Eric de Dampierre (éd.), Une esthétique perdue. Paris : Presses de l'Ecole Normale Supérieure : 99-208. 
HORNBOSTEL Erich von, 1928, « African Negro Music », African Journal of the International Institute of African Languages and Cultures I/1.

SPERANZA Gaetano, 1995, « Sculpter au singulier » ", in : Eric de Dampierre (éd.), Une esthétique perdue. Paris : Presses de l'Ecole Normale Supérieure : 25-53.

VOISIN Frédéric et France CLOAREC-HEISS, 1995, «Echelles musicales et données linguistiques : vers une histoire des sociétés oubanguiennes », in : Ndroje Balendro : musiques, terrains et disciplines. Paris : SELAF : 119-139.

\section{NOTES}

1. On en trouvera de nombreuses illustrations dans G. Speranza (1995 : 36-53).

2. Pour de plus amples informations, il est nécessaire de se reporter à l'ouvrage précédemment cité dans lequel Marc Chemillier note (page 114) une information donnée par l'un des harpistes : «la cellule rythmique asymétrique était le propre des airs de danse nakùlúngù chantés par les femmes, et c'est seulement dans un passé récent qu'elle a été généralisée à d'autres danses, dont le gitangi ».

3. Simha Arom (1985) avait déjà signalé «Quant à nous, le seul type d'échelle que nous ayons rencontré présentant cette caractéristique provient de la population Nzakara. Il s'agit du système équihexatonique suivant: Si, Ré bémol, Mi bémol, Fa, Sol, La, Si. C'est, en d'autres termes, une 'gamme à tons entiers', utilisée pour un répertoire de chants féminins. Ici aussi, ce système côtoyait le système pentatonique traditionnel, qui était toutefois le plus couramment usité dans cette population. » De plus Frédéric Voisin note $(1995: 128 / 1)$ : « un tel accord ne serait donc pas équihexatonique défectif, comme on pourrait le penser, mais bel et bien équipentatonique au sens banda du terme [...] l'échelle conceptuelle procède par la sommation d'intervalles égaux sans faire appel au cadre préalable de l'octave. »

4. Instrument que l'on a déjà pu entendre dans un disque de la même collection: République Centrafricaine. Musique pour xylophones, enregistrements, texte et illustrations de Vincent Dehoux, un CD, LDX 274932.

5. «Chez les Nzakara, les lames ont une épaisseur deux fois moindre que celles des lames de xylophone Banda et Gbaya - mais d'une texture plus dense », (Voisin 1995 : 123). 